ÉGYPTE

monde arabe

\section{Égypte/Monde arabe}

$17 \mid 1994$

Soudan 2

\title{
L'Annuaire de l'Umma et la question sécuritaire
}

\section{Baudouin Dupret}

\section{Q OpenEdition}

\section{Journals}

Édition électronique

URL : https://journals.openedition.org/ema/1459

DOI : 10.4000/ema.1459

ISSN : 2090-7273

\section{Éditeur}

CEDEJ - Centre d'études et de documentation économiques juridiques et sociales

\section{Édition imprimée}

Date de publication : 31 mars 1994

Pagination : 157-183

ISSN : 1110-5097

\section{Référence électronique}

Baudouin Dupret, "L'Annuaire de l'Umma et la question sécuritaire », Égypte/Monde arabe [En ligne], 17 | 1994, mis en ligne le 08 juillet 2008, consulté le 07 juillet 2022. URL : http://journals.openedition.org/ ema/1459; DOI : https://doi.org/10.4000/ema.1459

Ce document a été généré automatiquement le 7 juillet 2022.

Tous droits réservés 


\section{L'Annuaire de l'Umma et la question sécuritaire}

\section{Baudouin Dupret}

1 Il y a un an, nous avons eu l'occasion, François Burgat et moi-même, de proposer aux lecteurs d'Égypte/Monde arabe la traduction d'un texte paru en introduction au premier numéro de l'Annuaire de l'Umma $a^{1}$. Il avait paru opportun, à ce moment, de faire état d'une publication constituant, en quelque sorte, le pendant au fameux Rapport stratégique arabe édité chaque année par le Centre d'études stratégiques du journal AlAhrâm. L'analyse du système politique égyptien que proposait l'historien et juriste Târiq al-Bichri semblait en outre particulièrement intéressante, dans la mesure où elle jetait une lumière originale sur ses dysfonctionnements.

2 Un an plus tard, le deuxième tome de cet Annuaire est paru². Sous la direction de Mahmûd 'Abd Allah 'Akif, avec pour consultants principaux Muhammad 'Imara, Kamâl al-Manûf, Sayyid Disûqî et Abû Bakr al-Mitwalli³, le nouvel Annuaire propose une analyse du système international (les Etats-Unis et le nouvel ordre international ; la région arabe dans la stratégie américaine après la guerre du Golfe), une analyse du monde arabe (le conflit israélo-arabe, la démocratie dans le monde arabe), une étude sur la République arabe d'Égypte (affaires internationales et affaires intérieures), enfin une analyse économique.

3 La liste des collaborateurs de I'Annuaire surfit à en révéler la tendance politique. Il s'agit d'une publication émanant de milieux proches des Frères musulmans, même si, dans le cas de Muhammad 'Imara par exemple (comme dans celui de Târiq al-Bichrî il y a un an), il n'y a pas d'affiliation directe. Cette orientation islamiste de l'Annuaire de l'Umma en fait, à vrai dire, une lecture très intéressante, qui permet au lecteur, à tout le moins, «de se familiariser avec le regard que porte sur l'actualité politique une composante importante de l'opposition $»^{4} .{ }^{5} \mathrm{Si}$, en effet, l'on sait que la presse a bénéficié, en Égypte, d'une politique d'ouverture, ce que l'on connaît moins, ce sont les contraintes qui affectent les organes de l'opposition. Les quotidiens al-Cha'b, al-Nûr, al-Haqîqa, alMukhtâr al-islâmî - pour ne prendre que ces exemples - qui sont acquis à la tendance islamiste, ont bien des difficultés à la représenter, tant l'accès aux médias s'avère 
sélectif. S'agissant du monde de l'édition, les contraintes ne s'avèrent pas moindres. La censure constitue une réalité difficilement contournable. Si elle prend souvent une coloration "néo-fondamentaliste " - pour reprendre les termes d'Olivier Roy ${ }^{6}$ - et frappe des ouvrages qui, aux yeux de establishment azhariste, portent atteinte à l'islam ${ }^{7}$, elle vise aussi les publications en provenance de l'opposition politique ${ }^{8}$ ou de milieux orientalistes développant une analyse faisant peu de concessions au régime ${ }^{9}$. Il est bon de souligner, à cet égard, que l'Annuaire de l'Umma semble avoir fait, lui aussi, les frais d'une mesure de censure. Il était en tout cas impossible, peu après la parution de l'ouvrage, de se le procurer dans quelque librairie que ce soit.

4 L'extrait de I'Annuaire de l'Umma dont nous proposons ci-après la traduction se révèle intéressant à d'autres égards. Il constitue, en tout premier lieu, une sorte de dossier de presse. L'information est, en effet, tirée pour l'essentiel des principaux quotidiens égyptiens, officiels ou d'opposition. Le thème de ce texte est la politique sécuritaire. C'est là son second intérêt, dans le contexte d'une actualité brûlante. Il nous montre d'abord que la question ne date pas de ces derniers mois, mais qu'elle remonte certainement aux débuts du second mandat du président Moubarak ${ }^{10}$. Il met ensuite en relation - comme l'indique le sous-titre - sécurité politique et sécurité publique (ou sociétale), cette dernière n'étant toutefois envisagée que sous le seul angle d'une agression sexuelle survenue sur l'une des principales places du Caire. Si pareille association mène inévitablement l'analyste à relever la tendance quelque peu puritaine de l'auteur du texte, autant préoccupé de la préservation des valeurs morales de la société - même s'il se complaît moins dans les détails de cette affaire de mœurs que ne l'ont fait la plupart des organes de presse - que de la dégradation du jeu politique, il convient toutefois de souligner que l'étude repose sur certains chiffres intéressants. Elle suggère aussi l'abandon par l'État d'un ensemble de responsabilités ayant trait à l'économie ou aux relations et services sociaux ${ }^{1112}$. Troisième intérêt de ce texte : il ne relie pas nécessairement la violence politique et les mouvements islamistes, glissement que ne manque pas d'opérer, pour sa part, le Rapport stratégique.

5 Cette dernière remarque permettra de conclure cette introduction en soulignant combien serait utile la comparaison de ces deux publications majeures que sont le Rapport stratégique et l'Annuaire de l'Umma. Certes, le Rapport conserve la vedette dans ce genre de production. Certaines faiblesses ne manquent toutefois pas d'apparaitre aussi bien dans la méthode que dans le contenu et le traitement des données, faiblesses que l'Annuaire, sans pouvoir prétendre remplacer le Rapport, tend à partiellement compenser.

\section{La politique sécuritaire et la problématique de la relation entre sécurité politique et sécurité publique}

6 L'Égypte a été la proie, ces dernières années, de toutes sortes de méfaits sociaux, au nombre desquels les sanglants affrontements entre les forces de sécurité et certains membres des groupes organisés s'opposant au régime. Le phénomène s'est répété dans plusieurs régions du pays, et particulièrement dans le Sa'îd, aussi les efforts de nature sécuritaire se sont-ils portés principalement sur l'élimination des périls menaçant la sécurité du régime. Ceci a conduit certains analystes à dire que les services de sécurité égyptiens faisaient passer la sécurité politique du régime en place bien avant la sécurité publique. 
7 Pour bien saisir la nature de la relation qu'entretiennent sécurité politique et sécurité publique, telle qu'elle ressort de différents cas concrets, l'on peut mettre l'accent sur différents points fondamentaux :

1. En dépit du fait que le budget du ministère de l'Intérieur ait dépassé, d'après les chiffres officiels avancés pour l'exercice 1990-91, l'ensemble des crédits alloués à la santé et à l'enseignement réunis, les rapports de la Sécurité générale révèlent l'existence d'une disproportion entre le nombre de citoyens et la protection destinée à assurer leur sécurité. Tandis que pour l'année 1985, l'on comptait un agent des services de la sécurité pour 241 citoyens, l'on est passé au chiffre d'un agent pour 328 citoyens en 1990. Pendant cette même période, le total des effectifs de la Sécurité, composés - à partir d'investigations sommaires de policiers, gardes et soldats, est passé de 194. 044 hommes, en 1985, à 164. 046 en 1990. Il n'est, dès lors, pas possible d'affirmer que la croissance démographique est la cause première de la réduction des moyens mobilisés pour assurer la sécurité.

2. Cette réduction des moyens d'intervention sécuritaires s'est accompagnée d'une augmentation du taux de criminalité en Égypte au cours des cinq dernières années. Les crimes passibles de répression pénale sont passés de 2019 à 2406 en 1990. La seule ville du Caire a vu leur nombre passer de 388 en 1985 à 500 en 1990.

3. Les rapports publiés par les organisations de défense des droits de l'homme ont, d'un autre côté, fait état des sévices commis par les forces de sécurité contre les citoyens. Ainsi, par exemple, le rapport d'Amnesty International a-t-il mentionné que «la torture des prisonniers politiques n'a pas connu de répit en Égypte depuis l'instauration, il y a dix ans en octobre 1981 - de l'état d'urgence. La convention prohibant la torture pratiquée dans le but d'obtenir rapidement des aveux n'a, pour sa part, pas été respectée. Par ailleurs, les responsables de la torture n'ont pas eu à répondre de leurs actes. »

Le rapport d'Amnesty international de 1992 confirme la poursuite des atteintes portées aux droits de l'homme en Égypte, au titre desquelles la torture, les meurtres, les disparitions, les arrestations arbitraires et la prise en otage ' des familles [de personnes recherchées]. Le rapport mentionne également la poursuite de la répression policière (Rapport d'Amnesty international, 1992, p. 250 ss.).

Dans le même ordre d'idées, le rapport de 1990 de l'OADH (Organisation arabe des droits de l'homme) établit que le nombre de détenus, du $1^{\mathrm{er}}$ mars 1986 au 9 février 1989, s'est élevé à 12472 et que la Haute Cour de sûreté de l'État a décidé, après examen des plaintes des prisonniers, l'élargissement de 12448 individus. Le ministère de l'Intérieur a interjeté appel pour 9243 de ces décisions, ce qui équivaut à $70 \%$ des décisions d'élargissement.'

10 Comme l'observe cette même organisation, le phénomène de la détention d'otages s'est banalisé en tant que pratique sécuritaire. Ces otages sont choisis parmi les proches de la personne recherchée et restent détenus jusqu'à ce que cette dernière se livre à la police.

11 Les rapports de l'OEDH (Organisation égyptienne des droits de l'homme) observent l'extension de l'utilisation de l'appareil sécuritaire à des fins de répression aveugle, comme l'atteste la survenue d'incidents ayant fait plusieurs dizaines de morts. Cette organisation observe également que la police utilise couramment contre des citoyens la technique de la punition collective (Rapport de l'OEDH, année 1990; Al-Cha'b, 19 mai 1992).

12 Il vaut également la peine de mentionner que le ministère de l'Intérieur a refusé d'admettre ce qui a été établi dans les rapports des différentes organisations de défense 
des droits de l'homme. Il a également démenti ne se préoccuper que des seuls faits relevant de la sécurité politique. Le ministre de l'Intérieur a déclaré qu'il tenait à ce que «soit effective la présence des forces de sécurité dans les rues et sur les places des grandes villes ». De fait, il a inauguré, au début de 1992, le système des «forces d'intervention rapides " : de nouvelles voitures de police embarquent des équipes de 4 à 6 policiers sous la direction d'un officier. Les armes dont disposent ces forces sont suffisantes pour leur permettre d'empêcher que des crimes ne soient perpétrés et d'assurer la sécurité dans les grandes villes égyptiennes.

Dans cette section de notre rapport, nous allons nous concentrer sur deux événements principaux, l'affaire Salsabîl et celle de 'Ataba. Toutes deux illustrent sans ambiguïté la problématique de la relation qui unit, dans l'actuelle politique sécuritaire égyptienne, la sécurité politique (première affaire) et la sécurité publique (seconde affaire).

Au début du mois de lévrier 1992, les forces de sécurité investissent le siège de la société informatique Salsabîl et en arrêtent les propriétaires, accusés d'appartenir à une organisation secrète cherchant à prendre le pouvoir et d'entretenir des relations avec l'association des Frères musulmans (Al-Ahrâm, 10 février 1992).

Selon diverses révélations provenant du ministère de l'Intérieur et du procureur de l'État après l'arrestation des inculpés, la société Salsabîl aurait eu de véritables objectifs politiques et aurait visé, sous son couvert, le cœur du régime. Ce à quoi la partie défenderesse réplique que les accusations formulées à l'endroit des propriétaires de la société manquent de preuves et que les « révélations » du procureur sont totalement infondées. Elle tiendra ensuite - si l'on en croit les indications de Mukhtar Nûh, l'un de ses membres - une conférence de presse dans les locaux de la branche cairote du Syndicat des avocats, le ministère étant passé de l'investigation à l'accusation des trois inculpés, lesquels ont été incarcérés avant que l'instruction proprement dite n'ait débuté, en raison de leur implication dans l'affaire 87 de la sécurité de l'État, rôle de 1992 (Al-Cha'b, 17 mars 1992).

Plusieurs remarques s'imposent pour évoquer cette affaire avec précision et en faire ressortir les tenants et aboutissants :

1. Les affaires traitées par la société Salsabîl ne concernaient pas exclusivement le secteur privé. Bien au contraire, environ $40 \%$ de l'ensemble de ses transactions étaient destinées à des organismes relevant du ministère égyptien de la Défense. Ce dernier a traité avec Salsabîl en adoptant un point de vue national et en la considérant comme une société nationale offrant d'excellents services. Les activités de cette société, qui avait pignon sur rue, sont donc devenues peu à peu florissantes, notamment quand elle est entrée dans la procédure d'adjudication organisée par l'administration des derniers Jeux panafricains au Caire. Et cela, même si une autre société a emporté l'adjudication, en dépit de l'importante différence entre les prix proposés par Salsabîl et ceux pratiqués par ses concurrents (alCha'b, 17 mars 1992).

2. Il n'y avait pas lieu de craindre l'orientation islamique des propriétaires de cette société, qui s'était spécialisée dans le secteur de l'informatique. Mais .c'était là une question sensible qui suscitait inévitablement la colère du pouvoir en place, engagé dans une confrontation sans précédent avec les groupes islamistes armés pour mettre fin à l'usage de la violence contre les forces et les symboles du régime en place.

3. cLes informations concernant la structure Salsabîl sont intervenues au moment même où l'on procédait au démantèlement d'une cellule d'espionnage israélienne dirigée par l'agent Fâris Misrâtî et sa fille Fâ'iqa Misrâtî. Cela a-t-il échauffé les esprits plus que de raison ou n'y avait-il là ; pour les services de sécurité égyptiens, qu'une coïncidence? 
17 De profonds différends ont opposé, dans cette affaire, la partie défenderesse et les enquêteurs. Selon Mukhtâr Nûh, les différentes mesures adoptées contre les inculpés investigations, arrestation puis incarcération - l'ont été en vertu de la loi d'urgence. Toujours selon lui, la partie défenderesse avait clairement fait connaitre son opposition aux parquets de la sécurité de l'État, ces derniers ne concourant pas au bon déroulement des investigations. Il dit avoir présenté au procureur général une note en ce sens, après le refus du parquet de transmettre à la défense le mémoire des faits et le permis de contrôler et d'inspecter (al-Cha'b, 17 mars 92).

Il n'est pas sans intérêt de savoir que le ministre de l'Intérieur [à l'époque 'Abd alHalîm Mûsâ, ndli]- a affirmé, après l'arrestation des propriétaires de la société, que la Sécurité s'était conformée à l'ensemble des procédures légales dans les opérations de surveillance des accusés et qu'il s'était contenté d'apposer les scellés au siège de la société, où se trouvait l'ensemble des pièces et documents, jusqu'à ce que le parquet se rendît sur les lieux et classât ces pièces lui-même (al-Jumhûriyya, 19 février 1992).

Parmi les questions les plus épineuses, il faut rappeler que la partie défenderesse a non seulement été privée, au début, de la communication du mémoire détaillant les accusations dont faisaient l'objet les propriétaires de Salsabîl, mais que, surtout, elle s'est vue exclue de toute participation aux travaux du comité d'analyse et de dépouillement des disquettes informatiques appartenant à la société. Elle a par conséquent invoqué la nullité du rapport établi par ce comité au motif qu'il avait œuvré en l'absence de la défense et du parquet général, et aussi parce que le comité comprenait en son sein un officier de la police bien que le ministère de l'Intérieur fût partie à l'affaire (al-Cha'b, 3 juin 1992).

20 L'examen de l'affaire Salsabîl et de ses développements tend à montrer que les institutions et les forces politiques ayant manifesté le plus grand intérêt à la question sont la Sécurité, représentée par le ministère de l'Intérieur, le Parti du Travail et l'Association des Frères musulmans. C'est parfaitement logique et naturel puisque la Sécurité a accusé publiquement et clairement ces derniers de financer et de soutenir la société Salsabîl. Les autres forces politiques ne semblent pas avoir eu, quant à elles, d'intérêt direct dans l'affaire.

\section{La position du ministère de l'Intérieur}

21 Celle-ci peut s'articuler autour des éléments suivants :

1.-Le «gonflement» médiatique insistant sur l'importance de la découverte d'une 'organisation' Salsabîl visant à attenter à la sûreté de l'État : le ministre de l'Intérieur, 'Abd al-Halîm Mûsâ, annonce à plusieurs reprises que les pièces saisies au siège de la société, tout comme les aveux des trois accusés arrêtés à ce même siège, confirment que les membres de cette organisation, nombreux au demeurant, visent le cœur même de l'État; l'on aurait même découvert un document faisant état de la composition du futur gouvernement censé prendre les commandes de l'État en cas de succès de l'insurrection (Al-Ahrâm, $1^{\mathrm{er}}$ février 1992).

2. Le ministre de l'Intérieur accuse explicitement les Frères musulmans de se trouver derrière la société Salsabîl et qualifie cette dernière d'«émanation » de l'Association des Frères musulmans, ces derniers entretenant des liens étroits avec les sociétés, de placement de fonds et les organisations de défense des droits de l'homme. Il affirme que la société Salsabîl n'est pas une cellule d'espionnage mais bien une cellule cherchant à attenter à la sûreté de l'État ; que l'opération ne vise pas seulement à réunir des informations ; enfin, que les Frères 
musulmans sont libres d'infirmer ou de confirmer. " Nous parlons », déclare-t-il alors, « des pièces que nous avons sous les yeux. Et je confirme qu'il existe d'étroites relations entre les propriétaires de Salsabîl et les Frères, et que certains d'entre eux ont participé à la création de la société. » (Rûz al-Yûsuf, 24 février 1992)

3. L'opposition politique entre les services de sécurité et les propriétaires de la société Salsabîl est manifeste. Le 13 mars 1992, le ministre élève encore d'un cran ses attaques contre les accusés, répétant que l'organisation Salsabîl constitue la source d'inspiration des extrémistes et que ses membres mettent la science et la technologie au service de leurs ambitions, couvrant ainsi leurs plans véritables. Le ministre va jusqu'à endosser le rôle de mufti en déclarant textuellement: «Je jure par Dieu que les membres de ces organisations n'ont aucun rapport avec la religion et n'en connaissent rien, mais servent leurs intérêts et leurs désirs personnels. » (Al-Jumhûriyya, 14 mars 1992)

4. Cette affaire confirme, l'importance accordée par l'appareil sécuritaire à la sécurité de l'élite politique au pouvoir - autrement dit, l'importance de ce qu'on appelle la sécurité politique bien avant la lutte contre la criminalité. Le ministre de l'Intérieur récuse cependant cette distinction et insiste sur le fait que « la sécurité a une signification globale qui embrasse les sécurités politique et publique. Toutes deux procèdent de l'essence même du travail de la Sécurité ». Ce faisant, il montre qu'il y a carence dans la préparation des forces de sécurité, ce qui le poussera à créer un nouvel institut pour les sous-officiers (al-Akhbâr, 22 avril 1992).

22 Ainsi, c'est l'empreinte du politique qui prédomine dans l'affaire Salsabîl, si l'on veut bien mettre en relation les informations en la matière avec la tentative de l'appareil sécuritaire de gagner en crédibilité et respectabilité, au beau milieu de la confrontation sanglante avec les associations islamiques violentes. Cela ressort clairement des propos du ministre de l'Intérieur, proclamant fièrement que "grâce à Dieu, la Sécurité égyptienne est rassortie grandie de cette affaire » (Al-Jumhûriyya, 14 mars 1992).

\section{La position de l'Association des Frères musulmans}

23 Ahmad al-Malat, délégué du Guide suprême des Frères musulmans, blâme tout d'abord le ministre de l'Intérieur, d'une part pour avoir fait incarcérer les propriétaires de la société, d'autre part pour les accusations qu'il a formulées à leur encontre (contacts avec l'extérieur, mise à profit de leurs ressources pour préparer l'insurrection visant l'État et collaboration avec des services étrangers).

24 Ahmad al-Malat dénonce également l'allusion du ministre à de prétendus liens secrets entre les propriétaires de la société et l'Association des Frères musulmans pour "préparer la prise du pouvoir ». Il s'adresse au ministre en ces termes: «Par Dieu, n'es-tu pas, à l'instant, étreint par le remords, d'avoir enfermé ces jeunes gens sans qu'ils n'aient commis d'autre faute que de croire en leur Seigneur, Lequel les a investis d'un savoir qui leur permet de rivaliser avec les plus grandes maisons dans le secteur de l'informatique? N'as-tu pas songé un instant que, par ton action, tu as fermé l'une des portes du savoir islamique, par lequel les musulmans peuvent rivaliser avec ceux qui ne le sont pas?» (al-Cha'b, 16 juin 1992)

Ahmad al-Malat insiste sur le fait que pareille attitude trouve son origine dans la crainte de tout ce qui est islamique. Il appelle à la mobilisation de toutes les forces disponibles pour faire face au danger. 


\section{La position du parti du Travail}

L'organe du parti du Travail, Al-Cha'b (Le Peuple), s'intéresse vivement à l'affaire Salsabîl, publiant aussi bien les informations concernant l'affaire et ses développements que les déclarations de la défense. D'après le journal, les accusations portées contre les propriétaires de la société n'ont pas l'ombre d'une preuve. Par ailleurs, les pièces et documents que-le ministre de l'Intérieur prétend avoir obtenus ne vont pas au-delà des quelques publications diffusées par les Frères musulmans et qui remplissent les librairies. Il s'agit en fait d'une tentative de monter l'affaire en épingle et d'y impliquer d'autres éléments, tels les syndicats professionnels et les associations d'enseignants des universités égyptiennes. Et le journal al-Cha'b d'insinuer que le ministère de l'Intérieur, sans aucun doute, cherche à étendre la confrontation à tous les éléments islamiques, quelles que soient leurs orientations.

Selon le Parti du Travail, l'affaire Salsabîl doit être considérée, comme un épisode dans le feuilleton de la confrontation globale que mène l'appareil sécuritaire contre les différents courants islamiques d'Égypte.

\section{L'affaire de 'Ataba} débouche sur l'amendement de l'article 268 du Code pénal : désormais, le crime de viol 
est puni de travaux forcés à perpétuité et non plus temporaires, la sentence pouvant même être la peine de mort dans certaines circonstances spécifiées par la loi (Al-Ittihâd, 5 avril 1992).

L'on peut, à l'examen de ce projet, le soupçonner de contrevenir à la loi islamique (charî'a) : il a en effet le défaut majeur de ne pas aborder la question de l'honneur et de ne pas infliger de peine pour l'acte de fornication par consentement (al-zinâ bi-l-tarâdf) (Al-Haqîaa, 18 avril 1992).

Le ministre de l'Intérieur ne cache pas son émotion et sa colère, en particulier après le scandale provoqué par la réduction des effectifs de la Sécurité et les allégations concernant sa négligence des dimensions sociétales du problème de la sécurité. Il en résulte des interventions pour le moins contradictoires :

1. Dans une brève déclaration devant l'Assemblée du Peuple le 22 mars, le ministre affirme que l'incident de 'Ataba fait partie de ces affaires banales comme il s'en produit dans tous les pays du monde. «La faute en incombe à la presse, qui a gonflé l'événement et lui a donné une importance excessive. C'est une affaire banale dans une ville de 15 millions d'âmes, dans un pays comme l'Égypte dont la population dépasse les 57 millions d'habitants. » (Sabâh alKhayr, 26 mars 1992)

2. Il revient cependant sur ses propos quelques jours après, affirmant que l'incident de 'Ataba est « contraire à la nature de la société égyptienne », qui fait assaut de zèle dans le domaine des valeurs religieuses et morales. Un Egyptien, eh effet, ne peut que se conformer aux us et coutumes de son pays. "Ma déclaration devant l'Assemblée du Peuple ne visait pas à minimiser l'importance de l'incident, mais à faire la lumière sur certaines questions soulevées par quelques députés, en particulier la réduction du personnel de la Sécurité. » (alAkhbâr, 25 mars 1992)

3. Parmi les éléments montrant clairement que les déclarations du ministre contredisent les faits, il faut relever que selon le premier avocat général Hânî Khalîl, le commissaire de police qui a arrêté deux des accusés appartient au poste d'Héliopolis et se trouvait par hasard sur le chemin du travail. Il n'appartient pas au poste des inspecteurs du Mûskî, dans le secteur duquel s'est produit l'incident, contrairement à ce qu'a affirmé précédemment le ministre (al-Ahâlî, 25 mars 1992).

4. Cet incident soulève manifestement la question du lien entre sécurité politique et sécurité publique. Le ministre essaie de s'en tenir à l'unité du concept de sécurité. À la question que lui pose le quotidien al-Ahrâm - «Est-il vrai que nous nous préoccupons davantage de sécurité politique que de protection contre la criminalité ? Nous n'en voulons pour preuve que les dizaines de voitures de la Sécurité centrale (al-Amn al-matkazî) sillonnant les rues du Caire tandis que les seuls policiers en faction sont les conscrits qui règlent la circulation et gardent les bâtiments publics »-, le ministre répond: «Je ne connais rien qui s'appelle " sécurité politique ", et rien non plus qui s'appelle «protection contre la criminalité ». La sécurité n'a jamais été parcellisée. Ma fonction, c'est la sécurité nationale, la sécurité des biens de la nation, de son honneur, de sa respectabilité et de sa vie, la sécurité de l'État qui s'abrite sous son toit. Si nous ne jouissons pas d'une excellente réputation de pays sûr et calme, nous perdrons notre unique revenu et ne verrons plus un seul touriste. » (al-Ahrâm, 27 mars 1992) 


\section{La position des institutions religieuses officielles}

\section{Le chaykh Al-Azhar}

Selon ce dernier, le chaykh Gadd al-Haqq, s'exprimant dans un article paru dans AlAhrâm, l'incident de 'Ataba est " une infamie, une honte infligée à la société islamique en général et à la société égyptienne en particulier. Il est inévitable que la conscience des hommes de toutes religions s'en émeuve ». Cet incident est à ranger, selon l'imam suprême, parmi les méfaits encourant la peine légale réservée à ceux qui s'en prennent à l'islam (hirâba). Il exige de signer la condamnation du coupable de cet acte déplorable, qui excède en gravité le rapt et le viol. Aux citoyens qui sont restés passifs lors de l'incident, il déclare: "Ceci s'inscrit dans le cadre du pouvoir discrétionnaire (ta'zîr) dont dispose le juge contre celui qui était en mesure de prêter secours à quelqu'un le lui demandant et qui s'en est désintéressé. Les témoins étaient sans nul doute en mesure de prêter assistance à la personne agressée. La peine discrétionnaire (hadd alta'zîr) entraîne, au minimum, l'amende ou l'emprisonnement" (Al-Ahrâm, 27 mars 1992).

\section{Le mufti de la République}

Celui-ci, le docteur Sayyid Tantâwî, réclame l'exécution publique des auteurs du crime de 'Ataba, « en guise d'avertissement pour tous ceux qui seraient tentés de commettre pareille horreur » (Al-Wafd, 29 mars 1992).

\section{Le ministre des Waqfs}

$\mathrm{Au}$ cours du meeting populaire tenu par ce dernier à Qawîsnâ, les ulémas présents réclament, contre les auteurs de l'incident de 'Ataba, l'application de la peine légale réservée à ceux qui s'en prennent à l'islam, qualifiant ces derniers de "pourriture ». Les participants au meeting en viennent également à blâmer la passivité des citoyens, «pathologie dangereuse»: il est indispensable de «débarrasser la société de ces perversions » (Al-Wafd, 25 mars 1992).

\section{La position des partis politiques}

39 Les principaux partis à s'être préoccupés de l'impact de l'affaire et à avoir proposé des solutions permettant d'y remédier sont le Parti du Travail (Hizb al-'amal), le Parti du Rassemblement (Hizb al-tajammu) et le Wafd.

\section{Le parti du Travail}

Par la voix de son organe de presse officiel, Al-Cha'b, ce parti affirme avec insistance que l'incident constitue « un crime hideux, une atteinte à la Nation ». La minimisation, par le ministre de l'Intérieur, de l'importance du crime, montre à quel point « l'enquête est faussée " et constitue une "offense aux victimes». Il y a là aussi une dégradation dangereuse de la sécurité. La responsabilité, selon le parti, en revient entièrement au régime en place. Ce dernier gouverne par la répression et la coercition, estime le journal, ce qui éloigne le citoyen de tout sentiment d'honneur, de courage ou d'adhésion et l'empêche de relever les défis posés par les abus qui sont le lot quotidien 
du pays. Al-Cha'b rejette l'entière responsabilité de l'événement sur le gouvernement Sidqî, qui a échoué dans sa tentative de remédier aux causes de la dégradation de la sécurité publique. Se contenter de renforcer les peines ne suffit pas, affirme le journal. Il faut au contraire traiter les problèmes sociétaux à la racine (Al-Cha'b, 7 avril 1992).

\section{Le parti du Rassemblement}

Selon lui, l'incident constitue l'expression de l'humiliation de l'homme et de la femme en général, l'expression de la dégradation des valeurs morales, voire même l'expression d'une répression enfermée dans sa propre logique. C'est là la preuve du manque d'institutions ou d'associations à même de résorber la colère populaire, tandis que la jeunesse se morfond, que l'on vit une crise du logement et de l'emploi et que toute dimension affective de l'existence est refoulée. Il faut, en conséquence, absolument " ouvrir un dialogue vaste et profond qui permette, par un surcroît de démocratie, d'infléchir le destin » (Al-Ahâlî, 8 avril 1992).

\section{Le Wafd}

Le Wafd se prononce pour un renforcement des peines afin de lutter contre les criminels et de garantir la paix de la société. Il revendique également l'exécution publique. Ce renforcement des peines n'est pas, aux yeux du Parti Wafd, en contradiction avec les lois divines (Al-Wafd, 19 mars 1992).

\section{La politique de la confrontation violente}

L'Égypte est actuellement le théâtre de confrontations multiples entre les forces de la sécurité et les éléments des groupes islamistes extrémistes. Les incidents sanglants qui ont eu lieu ces dernières années - qu'il s'agisse de ce que j'appellerai sédition confessionnelle (fitna tấifiyya) et pratique extrémiste (mumârasa al-tatarruf) ou de ce à quoi je référerai en tant qu'actes terroristes (a 'mal al-imâb) - ont non seulement ébranlé la .stabilité politique du pays mais aussi fragilisé la structure du corps social égyptien. L'on peut saisir les traits et les composantes de la politique sécuritaire égyptienne dans sa façon de traiter le phénomène, en exposant les incidents d'Imbâba, de Dumyât, du Fayyûm, de Dayrût, de Banî Suwayf et enfin l'assassinat de Farag Fawda.

\section{Les incidents d'Imbâba}

Ils éclatent à la fin du mois de septembre 1991. Il s'agit au départ d'une simple querelle opposant deux familles, l'une chrétienne, l'autre musulmane, querelle qui s'envenime après l'intervention de personnes extérieures prenant fait et cause pour l'une ou l'autre partie. Il en résulte nombre de blessés et la mise à sac de deux églises et d'une quarantaine d'échoppes (Rûz al-Yûsuf, 30 septembre 1991).

À la suite de ces incidents, les forces de sécurité interpellent environ 600 suspects et incarcèrent près de 60 individus. Elles ratissent la région pour mettre la main sur les membres des groupes islamistes extrémistes (jamâ'ât islâmiyya mutatarrifa) dans les secteurs de Bachtîl, Munîra, Gharbiyyâ, Bulâq al-Dakrûr et Haram (Al-Cha'b, 24 septembre 1991). 
lissement vers la violence accentue la confusion entre, d'une part, ces groupes islamistes et, de l'autre, la Sécurité qui fait usage de méthodes violentes dans la confrontation et ne respecte pas les règles conditionnant l'emploi des moyens de coercition dont elle dispose. Le quartier d'Imbâba vivra d'ailleurs deux fois en sept mois de pareils événements: début mai 1992, des heurts opposent certains membres de l'organisation du Jihâd aux forces de sécurité : deux militants de l'organisation sont abattus et une fillette de onze ans blessée.

Ces événements sont ensuite exploités dans deux perspectives différentes. La première met l'accent sur la question de la sédition confessionnelle et de l'atteinte à l'unité constitutive de Umma, la seconde visant à susciter une campagne de soutien aux forces de la sécurité et à l'arrestation des membres des groupes islamistes violents.

\section{La réaction du pouvoir exécutif}

Après les incidents d'Imbâba, le ministre de l'Intérieur affirme que pareils événements peuvent se produire « en tout lieu et en tout temps' et qu'il ne s'agit que d'une « simple querelle ». Il n'y a pas lieu, selon lui, de parler de sédition confessionnelle. Il annonce en outre l'arrestation de 32 personnes (de jeunes extrémistes et des délinquants) ayant essayé de tirer parti des incidents (Al-Ahrâm, 24 septembre 1991).

Le gouvernement tient ensuite à Imbâba un meeting populaire auquel participent le ministre des Waqf, le ministre des Pouvoirs publics, le gouverneur de Gîza ainsi que des représentants d'al-Azhar et du pape Chenuda. Le rassemblement s'achève sur le constat suivant : les incidents d'Imbâba ne sont que des situations isolées, sans relation aucune avec une quelconque sédition confessionnelle. L'on préconise de créer un comité national permanent comprenant des ulémas, des intellectuels et des sages musulmans et chrétiens ainsi que des représentants du Haut Conseil des affaires islamiques, d'alAzhar et de l'Église " pour étudier toutes les mesures susceptibles d'éviter l'éclatement de l'unité nationale » (Al-Ahrâm, 8 octobre 1991).

\section{Le parti Wafd}

Il a tendance à considérer les incidents d'Imbâba comme des cas isolés sans lien avec la question de la sédition confessionnelle (Al-Wafd, 8 octobre 1991).

51

Lors d'une conférence intitulée "Sionisme et sédition confessionnelle », organisée par le comité religieux du Wafd, l'on évoque le rôle occulte joué par la colonisation et le sionisme pour tenter d'enraciner la sédition confessionnelle en Égypte (Al-Wafd, 29 octobre 1991).

\section{Le parti du Travail}

Il s'en prend, par la voix de son quotidien Al-Cha'b, à l'activité sécuritaire qui a été déployée pour faire face aux événements d'Imbâba. 'Adil Husayn souligne que

"les incidents séditieux se sont répétés et accélérés ces derniers mois, et ce de manière inhabituelle. Toute personne qui suit ce qui se passe chez nous ne peut qu'être confortée dans la conviction que le rôle joué par les étrangers, et les sionistes en premier lieu, est bien réel. Le Parti du Travail lutte avec détermination et sincérité pour déjouer la sédition confessionnelle et l'étouffer dans l'œuf, notamment au niveau des comportements quotidiens. Nos convictions islamiques et nationalistes nous y appellent. Si nous avons été à la tête de ceux qui se sont 
insurgés contre la colonisation et ses intrigues, comment pourrions-nous permettre aux complots fomentés à l'extérieur de semer le doute dans les rangs de la Nation? » (Al-Cha'b, 24 septembre 1991)

\section{Le parti du Rassemblement}

Pour Rif'at al-Sa'îd, secrétaire du comité central du parti, la pauvreté, le chômage et l'habitat informel - ce que l'on appelle la « ceinture de misère »- sont le détonateur de l'extrémisme. Le microbe, qui vient de l'extérieur, pénètre dans un corps affaibli que rongent la pauvreté et le malaise provoqués par le chômage.

Rif'at al-Sa'îd rejette l'entière responsabilité de la situation sur le gouvernement, du fait, entre autres, de la façon dont ce dernier conçoit les informations, « renforçant les extrémistes dans leur conviction d'être plus religieux que nous ». De même, le pouvoir est responsable d'avoir favorisé l'existence des groupes extrémistes pour porter atteinte à la gauche nationaliste, tout comme il est responsable des programmes d'enseignement qui établissent une distinction entre le musulman et le copte.

Le secrétaire du parti propose enfin que le président de la République visite Imbâba et inaugure la reconstruction de l'église incendiée, ne serait-ce que parce qu'elle est reconstruite aux frais du gouvernement (Al-Ahâlî, octobre 1991).

\section{Les Libéraux}

Selon ces derniers, la Sécurité a saisi le prétexte de la sédition confessionnelle pour s'en prendre au mouvement islamiste et l'épurer. Un journaliste d'Al-Haqîqa affirme que la Sécurité échauffe les médias à chaque incident, avant d'arrêter des jeunes gens dont elle prétend qu'ils fomentent la sédition confessionnelle et sapent l'unité nationale (AlHaqîaa, 28 septembre 1991). D'après ce journaliste, les Libéraux inclinent à interpréter en termes de « complot » les événements d'Imbâba et ceux qui leur sont apparentés : la Sécurité comploterait pour porter atteinte au mouvement islamiste.

\section{Les Frères musulmans}

Pour Mustafâ Machhûr, les accrochages entre musulmans et coptes sont un phénomène anormal, apparu seulement ces dernières années, et celui qui examine attentivement les événements peut en percevoir la dimension artificielle.

Il est du devoir des hommes de la Sécurité, estime-t-il, d'interdire la provocation pour se donner la possibilité de résoudre le problème. Leur insouciance provoque le soupçon ; tout se passe comme s'ils avaient poussé à la sédition parce que l'Amérique et l'ennemi sioniste en avaient ainsi décidé.

Ces événements, ajoute-t-il, surviennent généralement lors d'occasions politiques comme les élections, pour tenter de ternir l'image de l'islam et des Frères, quand bien même les Frères ont toujours été un facteur de sécurité et d'apaisement. Enfin, les Frères encouragent ceux qui appellent à la recherche, sur des bases scientifiques rigoureuses, de solutions justes. C'est en effet un devoir religieux et national (Al-Cha'b, $1^{\mathrm{er}}$ octobre 1991). 


\section{Les événements de Dumyât}

60 Vers la fin décembre 1991, des jeunes appartenant à la mouvance islamiste saccagent des cafés et arrachent des affiches de cinéma jugées indécentes (Al-Ahâlî, 2 janvier 1992). La Sécurité ne parvient pas immédiatement à les appréhender. Par la suite, l'officier Mutâwa' Abû-I-Najâ, nommé responsable des " affaires religieuses » à la Sécurité de Dumyât, est chargé d'arrêter les suspects, de les incarcérer et de les torturer (Al-Cha'b, $1^{\text {er }}$ janvier 1992).

En guise de représailles, de jeunes islamistes prennent d'assaut l'appartement de l'officier qui, les ayant vu arriver, ouvre le feu, faisant deux victimes parmi les assaillants. Les forces de la sécurité, à la suite de cet incident, lancent des campagnes d'arrestations à Dumyât; de nombreux jeunes quittent alors le gouvernorat. Trois suspects se réfugient dans un immeuble du village de Busârata, près de Dumyât. Les ayant découverts, les forces de la Sécurité assiègent l'immeuble et les liquident (AlAhâlî, 2 janvier 1992 et Al-Cha'b, $1^{\text {er }}$ janvier 1992).

Ce qui est vraiment caractéristique de l'incident, c'est que, malgré la mort dé trois jeunes suspects, les institutions officielles n'y prêtent aucune attention. Le ministre de l'Intérieur se borne à reconnaître, lors d'une conférence organisée dans le cadre de la Foire internationale du livre au Caire, que la Sécurité a procédé à l'élimination de trois jeunes gens qui tentaient de lui résister (Al-Ahrâm, 26 décembre 1991).

\section{Le Wafd}

63 Le parti affirme, par la voix de son organe de presse, que les trois victimes étaient des " extrémistes », tombés lors du combat les ayant opposés à la Sécurité. Il ajoute que le but des extrémistes était de terroriser la population locale et d'en découdre avec les officiers de police (Al-Wafd, 26 décembre 1991).

\section{Le parti du Travail}

Pour ce dernier, le meurtre des trois jeunes gens s'inscrit dans le cadre de l'élimination des islamistes organisée par le pouvoir. Les victimes étaient innocentes et aucun combat n'a eu lieu entre eux et la Sûreté, pour la bonne raison qu'ils n'avaient pas d'armes. Le journal Al-Cha'b tient ce qui s'est passé pour un « crime odieux » à ajouter au sombre dossier du ministère de l'Intérieur dans sa lutte brutale contre les islamistes (Al-Cha'b, $1^{\mathrm{er}}$ janvier 1992).

\section{Le parti du Rassemblement}

65 Il fait porter la responsabilité des incidents de Dumyât sur la jeunesse islamiste. Il affirme que les extrémistes s'en sont pris à l'officier de police et terrorisaient la population, tandis qu'eux-mêmes ont dévié des enseignements de l'islam (Al-Ahâlî, 2 janvier 1992).

\section{Les événements du Fayoum}

Début mars 1992, l'assassinat d'Ahmad 'Alâ' al-Dîn, officier responsable des 'affaires religieuses" à la Sécurité du Fayyûm, s'inscrit dans la série de confrontations sanglantes 
qui opposent la Sécurité au groupe des Chawqiyyîn, dirigé par l'émir Chawqî al-Chaykh (Al-Cha'b, $1^{\mathrm{er}}$ mars 1992).

L'on désigne le général 'Abd al-Wahhâb Hilâlî - qui, par la suite, devait aller à Asyût comme nouveau directeur de la Sûreté au Fayyûm. Il procède à des rafles étendues et à des arrestations aveugles, aboutissant à la capture de deux jeunes gens suspectés d'avoir commis l'assassinat (Al-Wafd, 23 mars 1992).

\section{La position du pouvoir exécutif}

Elle ressort du discours du $1^{\mathrm{er}}$ mai 1992 prononcé par le président de la République à l'occasion de la Fête du travail :

«Les balles du terrorisme qui frappent depuis des semaines et ont tué un officier de police qui accomplissait son devoir national, sont les balles de la haine qui visent la sécurité de l'Égypte et sa stabilité. Cette frange égarée dirige sa haine vers le cœur même de la société pour l'empêcher de se redresser et de se développer. Nous ne permettrons pas que les appels au mensonge et à l'ignorance menacent la stabilité de l'Égypte et sa sécurité, et fassent obstacle au destin et aux véritables objectifs de la Nation. En vertu de la loi et de la conscience du peuple, nous sommes en mesure de répliquer à cette frange qui a choisi le terrorisme comme doctrine et comme conduite. »

Par ailleurs, des divergences se font jour entre le leadership politique et l'appareil sécuritaire du gouvernorat du Fayoum quant à la façon de traiter ces événements. Alors que le gouverneur, M. Husayn Chahâta, souligne l'importance du dialogue entre les représentants du pouvoir et les membres de la Jamâ'a islamiyya, le directeur de la sécurité annonce que la police n'ouvrira aucune discussion avec le groupe extrémiste et répliquera à la violence par un surcroît de violence (Al-Cha’b, 17 mars 1992).

\section{Les institutions religieuses}

Bien qu'aucune institution religieuse n'ait écrit de rapport ou de communiqué sur les événements de Dumyât, les représentants de l'institution - le chaykh d'al-Azhar, le mufti et le ministre des Waqf - critiquent l'extrémisme et le recours à la violence ayant conduit à l'assassinat de l'officier de police du Fayoum. Dans un entretien accordé au journal Al-Akhbâr sur les événements de Dayrût et du Fayoum, le chaykh d'al-Azhar déclare: "Ceux qui s'engagent dans des actions violentes ne sont en aucun cas des musulmans. Ceux qui provoquent la haine et le mépris du régime ne peuvent être appelés les extrémistes de l'islam parce qu'ils ne professent pas l'islam et n'y appartiennent pas. Il faut étudier les causes qui les ont poussés à agir comme ils ont agi, sans aucune réflexion.. D'ailleurs, la Sécurité et les médias ont invoqué la nécessité de s'en tenir à la stricte vérité et à ne pas gonfler ces événements.» (Al-Akhbâr, 11 mai 1992)

71 Le ministre des Waqf, M. Muhammad Mahjûb, déclare, dans un meeting populaire organisé au Fayoum, que les Chawqiyyîn sont les tenants d'une pensée déviante et fomentent une sédition qui ne profite à personne. Ces gens, selon lui, sont coupables et sortis du groupe des musulmans (Al-Akhbâr, 17 mars 1992). Le ministre demande l'application de la peine légale réservée à ceux qui s'en prennent à l'islam (hirâba). L'Égypte n'est pas la demeure de la guerre (dâr al-harb) et n'a pas besoin d'aménager sa foi. C'est un pays pieux et la demeure de la paix (dâr al-salâm). Ses minarets rappellent jour et nuit l'unicité de Dieu (tawhîd) (Al-Wafd, 17 mars 1992). 
Quant au mufti de la République, ses propos sont des plus communs. Il rappelle que l'homme de la rue récuse la violence, qui incite à la haine, et qu'il n'est pas permis d'excommunier la communauté. "L'islam est contre l'extrémisme sous toutes ses formes. La voie du sang s'achève dans le sang. Nous sommes des prêcheurs, non des juges. Il nous incombe de réformer les esprits. » (Al-Akhbâr, 10 juillet 1992)

\section{Le Wafd}

73 L'officier de police tué au Fayoum est, selon le Wafd, à ranger au nombre des martyrs, et ses assassins les tenants d'une pensée extrémiste. Il rejette la responsabilité de l'événement sur le gouvernement pour ne pas s'être assuré le contrôle de ces régions éloignées, ce à quoi il faut ajouter l'inexistence de toute activité perceptible des partis politiques. La dégradation de la situation sociale est à l'origine des méfaits du terrorisme ; la colère monte au rythme de ce que la population entend sur ce qui se passe dans les prisons (Al-Wafd, 17 mars 1992).

74 Pour le Wafd, l'assassinat, qui provoque l'engrenage de la violence, n'est pas la solution. L'incident du Fayoum ne peut être distingué de la question de la violence dans la société, de la question des droits de l'homme, de la politique d'emprisonnements arbitraires ou de la nécessité de favoriser un climat démocratique véritable. À la démocratie, on ne peut opposer que le travail de l'ombre. Au dialogue, on ne peut opposer que la violence (Al-Wafd, 18 mars 1992).

\section{Le parti du Travail}

75 Il critique la violence et la contre-violence, qu'elles soient le fait des forces de police ou des groupuscules extrémistes. Le journal Al-Cha'b affirme que la violence ne provoque que sa réplique et ne laisse pas place à la réflexion. Le dialogue par la parole, non par les balles, est la solution. La politique sécuritaire en cours nous mène à la catastrophe, Dieu seul en connaît l'aboutissement (Al-Cha'b, 10 mars 1992).

Le Parti du Travail blâme également les forces de sécurité pour s'être livrées à des opérations de représailles après l'assassinat de l'officier, détruisant les maisons des suspects, imposant le couvre-feu et procédant à des emprisonnements arbitraires (AlCha'b, 17 mars 1992).

\section{Le parti du Rassemblement}

77 Selon le journal du parti, les extrémistes exploitent la crise économique et les agissements de la police pour susciter la sédition, alors que les Frères musulmans ont fait savoir leur refus de l'extrémisme et de la violence (Al-Ahâlî, 18 mars 1992).

78 Al-Ahalî demande, dans un communiqué, l'instauration du dialogue pour faire face à l'extrémisme de quelques groupes se revendiquant de l'islam politique. Il met en garde contre l'utilisation de la solution sécuritaire. Il faut nécessairement procéder à des aménagements dans les politiques économiques et sociales en cours, qui contribuent à l'émergence de l'extrémisme. Le communiqué demande l'arrêt des opérations sécuritaires qui provoquent les masses, comme la prise d'otages, les punitions collectives et la destruction du mobilier chez les suspects (Al-Ahâlî, 25 mars 1992). 


\section{Les Frères musulmans}

Pour 'Abd al-'Azîz 'Achrî, membre de la précédente Assemblée du Peuple et un des leaders des Frères au Fayoum, le fanatisme des groupes extrémistes trouve son origine dans le comportement de la police sous le régime de la loi d'urgence, dans l'incapacité du gouvernement à pourvoir le peuple du minimum vital (hadd al-kafâf) et dans l'absence de gestion des secteurs économique, politique, social et culturel (Al-Ahâlî, 25 mars 1992).

Il condamne l'assassinat de l'officier. Le dialogue par la raison et la logique est la seule solution. La violence ne peut amener que la violence. Le ministre de l'Intérieur doit respecter la loi jusqu'à ce que s'arrête ce « feuilleton » (Al-Cha'b, 17 mars 1992).

\section{Les événements de Dayrût}

81 L'intensité de la violence, la continuité des affrontements et les heurts armés qui ont caractérisé les événements de Dayrût, dans le gouvernorat d'Asyût, se sont développés sous l'effet de facteurs culturels, religieux et sociaux propres à la vie quotidienne du Sa'id égyptien. La première étape des événements sanglants de Dayrût advient le 9 mars 1992, suite à une altercation entre deux familles, l'une chrétienne, l'autre musulmane. Le différend porte sur la vente d'une maison. Comme souvent dans le Sa'îd, où les querelles prennent un tour aigu, il est fait usage d'armes à feu. Bilan : trois morts (un chrétien et deux musulmans) et cinq blessés des deux côtés.

Malgré l'enquête menée par le parquet et les mesures de sécurité draconiennes prises par le ministère de l'Intérieur dans le secteur, la confrontation reprend bientôt entre les deux factions. Certains éléments extrémistes islamistes s'en mêlent, ce qui aboutit à la mort de 13 personnes, toutes chrétiennes à l'exception d'une. Trois musulmans sont également blessés par balles ainsi que deux chrétiens, dont l'un mourra peu après.

La deuxième phase commence le 19 juin 1992 dans le village de Sanbû, lui aussi rattaché à la commune de Dayrût. Des heurts se produisent entre la Sécurité et certains membres de groupes islamistes violents (tels que le Jihâd), conduisant à la mort de 'Arafa Darwîch, l'émir du groupe. Trois autres individus sont blessés et huit suspects arrêtés.

écurité intensifie alors sa présence en envoyant de gros contingents (plusieurs milliers de soldats de la Sécurité centrale, équipés de blindés et d'automitrailleuses). De nombreuses opérations sont lancées contre les villages (Kûdiat al-lslâm, Banî Yahyâ ou Musâra, par exemple) servant de refuge aux islamistes. Des hélicoptères sont utilisés pour ratisser la région et poursuivre les militants dans leurs repaires (Al-Haqîqa, 27 juin 1992).

Enfin, la Sécurité impose le couvre-feu à Sanbû et Dayrût et ferme les routes principales et secondaires y menant. Malgré tout, les heurts entre les forces de police, les membres des groupes violents et certains habitants se poursuivent, s'apaisant parfois pour éclater de nouveau en d'autres endroits, comme les éruptions successives d'un volcan en pleine activité. 


\section{La position du pouvoir exécutif}

Ce dernier accorde toute son attention aux événements d'Asyût, eu égard aux dangers qu'ils comportent et à la violence particulière qui les caractérise. S'étant rendu à Asyût après la première phase des événements, le président Moubarak demande à la population de régler sans l'intervention de personne les problèmes qui peuvent survenir en son sein (Al-Jumhûriyya, 27 mai 1992).

À l'issue de la deuxième phase des événements, le président écarte, lors de propos tenus le 20 juin 1992, l'existence d'une ingérence étrangère qui, dans les événements de Dayrût, viserait à porter atteinte à l'islam. Il estime que les événements sont le fait d'éléments intérieurs (Al-Wafd, 21 juin 1992).

Quant au Premier ministre, M. 'Atif Sidqî, il déclare, lors d'un meeting populaire au Fayoum, que le gouvernement «ne restera pas les bras croisés devant quelque forme que ce soit de terrorisme ou d'extrémisme " et qu'il n'y aura "ni négligence ni hésitation dans la lutte contre toute tentative de mise à mal du principe de liberté politique » (Al-Ahrâm, 2 juin 1992).

Pour sa part, le ministre de l'Intérieur affirme, le 18 mars 1992, que les événements d'Asyût ne sont qu'une banale altercation entre deux familles et que seul le hasard a voulu que l'une d'elles soit chrétienne et l'autre musulmane (Al-Akhbâr, 19 mars 1992). Après les événements liés à la deuxième phase du conflit, il accuse certains éléments extrémistes d'être derrière la «boucherie » qui s'est soldée par la mort de 13 chrétiens (Al-Akhbâr, 16 mai 1992). Enfin, il annonce l'arrestation de tous les suspects membres de ces groupes, et affirme que la Sécurité maitrise la situation à Asyût et poursuivra jusque dans leurs bases les éléments étant à l'origine des troubles (Al-Ahrâm, 9 juillet 1992).

\section{Le parti du Travail}

- Celui-ci affirme, par l'intermédiaire de son organe Al-Cha'b, qu'on ne peut expliquer la tournure de "sédition confessionnelle " prise par les événements sans les réintégrer dans leur contexte. La question doit être mise en rapport avec la situation de «vide politique dans lequel nous vivons, le régime s'obstinant à interdire aux partis politiques l'exercice véritable de leur activité populaire, à opprimer la tendance islamiste, à manipuler les élections et à écarter les gens du processus décisionnel à l'intérieur du pays, à un moment où se multiplient les difficultés économiques et sociales. Tout ceci est à la source même des événements. » (Al-Cha'b, 12 mai 1992)

\section{Le Wafd}

91 Il fait de la dégradation des conditions économiques, sociales et sanitaires du village de Sanbû, ainsi que de l'indisponibilité des responsables - qui se consacrent exclusivement à leurs intérêts et à leurs problèmes personnels - le secret de l'explosion qui s'y est produite (Al-Wafd, 25 mai 1992).

92 Il diffuse une leçon publique sur la violence dans la société égyptienne. Le seul langage sécuritaire, précise-t-il, ne peut suffire à contrer la violence. Il faut absolument en rechercher les causes véritables (Al-Wafd, 21, 22 et 23 mai 1992). 


\section{Le parti du Rassemblement} gouvernement: "Le gouvernement est resté léthargique, indifférent et négligent jusqu'à ce que se produisent les heurts et se multiplient les incidents sanglants.» (Watanî, 5 juillet 1992) d'idées subversives en provenance de certains Etats de la région, qu'alimentent des informations déformées, un enseignement qui sème les germes de la haine et de l'animosité entre les individus d'un même peuple. Ceci est le fait d'autres pays de la région que Dieu a pourvus de richesses immenses qu'ils utilisent à financer ces groupes, de sorte qu'ils peuvent acheter dés armes et infiltrer les milieux ignorants dans le but de détruire l'Égypte et son régime. » (Watanî, 12 juillet 1992)

\section{Les événements de Banî Suwayf}

Au matin de 'Ayd al-fitr al-mubârak, des islamistes tentent de reprendre le contrôle de la mosquée al-Châdir, qui vient d'être rattachée au ministère des Waqfs. Les jeunes gens, munis d'armes et de pierres, s'enferment à l'intérieur de la mosquée, qu'assiègent alors les forces de la sécurité. Des coups de feu sont échangés, faisant quatre morts parmi les islamistes. Plusieurs dizaines d'entre eux sont arrêtés (Al-Wafd et Al-Ahrâm, 9 avril 1992).

La première réaction officielle à ces événements vient du général Ibrâhîm Sarhân, le directeur de la Sécurité à Banî Suwayf, affirmant qu'il n'y aura de dialogue avec les extrémistes que par des balles, qu'il leur appliquera « la peine légale réservée à ceux qui s'en prennent à l'islam» et qu'il ne leur laissera «aucune possibilité de s'exprimer » (Al-Ahâlî, 8 avril 1992 et Al-Wafd, 9 avril 1992).

Égypte/Monde arabe, 17 | 1994 
101 Pour le Wafd et le Parti du Rassemblement, c'est la Jamâ'a islâmiyya qui est à l'origine des incidents et des heurts avec la sécurité : n'est-elle pas en effet en possession d'armes, de cocktails Molotov, de poignards et de baïonnettes? (Al-Wafd, 4 avril 1992 et Al-Ahâlî, 8 avril 1992). On lit dans le quotidien Al-Wafd que « les jeunes causent un grave préjudice à l'islam en portant atteinte à la religion aussi bien qu'à eux-mêmes. Ils ne connaissent pas les limites de ce qui est licite au regard de l'islam. Ces combats portent préjudice au courant islamiste extrémiste comme à l'ensemble du courant religieux, modérés compris. Ils donnent au gouvernement le prétexte de la lutte contre le terrorisme pour justifier la réduction des libertés et la prolongation de l'état d'urgence." (Al-Wafd, 9 avril 1992) Quant au parti du Travail et aux Libéraux, ils qualifient ce qui s'est produit de «boucherie » visant à éradiquer les islamistes. Selon eux, la police aurait profité de la situation pour ouvrir le feu contre ces jeunes gens (AlCha'b, 7 avril 1992 ; Al-Haqîaa, 11 avril 1992).

\section{L'assassinat de Farag Fawda}

Le soir du lundi 8 juin 1992, Farag Fawda, connu pour ses orientations laïcistes, est abattu devant son bureau d'Héliopolis par deux individus circulant à vélomoteur. Son fils et l'un de ses gardes du corps sont blessés au cours de la fusillade.

Selon les communiqués du ministère de l'Intérieur, les deux personnes impliquées dans l'assassinat appartiennent à l'organisation islamiste al-Jihâd et résident à Zâwiya.alHamrâ. Toujours selon le ministère, l'assassinat de Fawda est intervenu en représailles à celui de Safwat 'Abd al-Ghanî, impliqué dans l'assassinat du président de l'Assemblée du Peuple, Rif'at al-Mahgûb (Al-Ahrâm, 21 juin 1992).

104 Le ministère adopte de sévères mesures de sécurité et organise une campagne d'arrestations - notamment de membres de la Jamâ'a islâmiyya - dans différents quartiers du Caire et de Gîza, les accusant d'implication ou de complicité dans l'assassinat de personnalités politiques importantes.

105 Si l'ensemble des courants politiques condamnent l'assassinat, ils divergent dans l'interprétation de ses tenants et aboutissants. La réaction la plus symbolique du pouvoir en place sera sans doute l'élaboration d'un projet de loi anti-terroriste, sous prétexte que les lois d'exception, toujours en vigueur en Égypte, ne suffisent pas à combattre les mouvements terroristes armés.

\section{Le pouvoir exécutif}

Immédiatement après l'incident, le président Moubarak déclare que le gouvernement prépare une loi anti-terroriste en lieu et place de la loi d'urgence, qui s'est révélée inefficace. Un comité d'experts est chargé de l'élaborer (Al-Wafd, 21 juin 1992).

De son côté, le Conseil des ministres s'accorde, début juillet, sur un projet d'amendement concernant certaines dispositions pénales et les lois sur les comptes secrets ainsi que sur les armes et munitions.

Si l'on en croit le ministre de l'Intérieur, la Sécurité savait que Farag Fawda était menacé par les extrémistes et s'était empressée, dès lors, d'assurer sa sécurité personnelle et de veiller sur son domicile et sur son bureau. Mais quelques semaines avant son assassinat, Fawda, que ces mesures exaspéraient, avait demandé avec insistance qu'elles soient levées (Al-Ahrâm, 21 juin 1992). 
109 Les médias officiels profitent de l'assassinat pour intensifier leur campagne d'opposition vis-à-vis des groupes extrémistes, les accusant d'être incapables de répondre à la pensée par la pensée et désignant leur recours au terrorisme et à la violence comme moyen de contrer ceux qui leur sont opposés. Ils exigent que l'on se hâte de promulguer une nouvelle loi sur le terrorisme.

\section{Le parti du Travail}

110 Ce dernier, selon son leader Ibrâhîm Shukrî, « condamne toute forme de violence. Ceux qui font usage de la force et des armes œuvrent contre nous. Les partis reconnus ne disposent que de la parole pour s'adresser aux gens. Nous ne cherchons pas à encourager le terrorisme. Cependant la violence et l'extrémisme sont aussi à rejeter quand ils sont le fait des éléments de la Sécurité. » (Al-Ahrâm, 6 juillet 1992)

De son côté, le journal Al-Cha'b publie des extraits des écrits de Farag Fawda concernant différents problèmes du monde arabe et islamique. Le bihebdomadaire rappelle que le militant laïciste appelait à une réconciliation politique, économique et humaine avec Israël, ainsi qu'à une invasion du Soudan par l'Égypte. Il montre les divers aspects - y compris les plus outranciers - du combat que menait Fawda contre les groupes islamiques, allant jusqu'à le,s accuser de stupre, les qualifiant de «tyranniques » et « obscurantistes » et affirmant qu'il n'était pas partisan du dialogue : «Pas de place au dialogue, pas plus qu'au jeu des questions-réponses. Il faut mener un combat total.» (Al-Cha'b, 16 juin 1992)

\section{Le Wafd}

112 Yâsîn Sirâg al-Dîn, une des plus symboliques figures du Wafd, affirme la nécessité d'une coalition de tous les partis et institutions démocratiques pour relever le défi du phénomène terroriste et l'éradiquer totalement. Il accuse le Parti du Travail et l'organisation "Jeune Égypte» d'encourager le terrorisme et l'extrémisme sous couvert de défendre les groupes islamistes, et de s'en prendre excessivement aux instances de la Sécurité, ce qui équivaut à soutenir implicitement la violence. Sirâg alDîn s'en prend également au Parti du Rassemblement qui, par la voix de son organe AlAhâlî, encouragerait la violence d'une autre manière, le journal exploitant l'événement avec de tels accents laïcistes qu'il provoquerait la susceptibilité et la colère des hommes de religion (Al-Ahrâm, 6 juillet 1992).

\section{Le parti du Rassemblement}

113 Ce dernier saisit l'occasion de l'assassinat de Fawda pour intensifier sa lutte contre les islamistes et les accuser d'être incapables de répondre à la pensée par la pensée. Le journal Al-Ahâli consacre une part importante de ses colonnes à des articles de partisans de Farag Fawda, " pionniers des Lumières » et " gens à l'esprit ouvert ", selon ses propres termes. Il publie également un communiqué du Comité national affirmant qu'il « récuse ce crime perpétré par les tenants de l'extrémisme et de la sédition confessionnelle » (Al-Ahâlî, juin 1992). 


\section{Les Frères musulmans}

114 L'Association des Frères musulmans déclare qu'elle regrette et condamne l'assassinat. Elle affirme son hostilité au terrorisme et estime que l'assassinat est l'arme de l'impuissant. Le courant islamique s'oppose à ces agissements. Il fait peser la responsabilité de l'incident sur les médias gouvernementaux, qui donnent aux laïcs et autres l'occasion d'exprimer leurs idées alors qu'ils en privent les islamistes (Al-Cha'b, 16 juin 1992).

\section{Conclusion}

Insister sur la distinction à opérer entre les événements liés à une sécurité qu'on pourrait qualifier de "politico-sociale » et les événements qui dépassent ce cadre et modifient la nature des actions sécuritaires conduit à dépasser le cadre étroit de cette question sécuritaire, qu'il s'agisse de sédition confessionnelle ou d'autres incidents à propos desquels on utilise les termes de "violence», d'" extrémisme», de "terrorisme». Ceci impose de ne pas se contenter d'une politique sécuritaire proportionnelle à l'importance des événements. Le récit des incidents relatifs à la "sédition confessionnelle", avec tout ce que cela signifie intellectuellement et culturellement et tout ce que cela implique pour les différentes composantes de la société et les fondements de leur coexistence - et bien que la politique sécuritaire l'ait ici emporté -, impose de prendre en considération tous les aspects culturels, intellectuels, sociaux et politiques des événements dans leur profondeur historique et de superposer ces événements. Ceci, on peut l'imaginer, permet une compréhension plus profonde et plus complète des phénomènes.

116 Discuter de ces événements et de leurs causes exige une analyse dont l'importance est confirmée par l'intérêt, exceptionnel, manifesté par les différents organes de presse, officiels ou d'opposition, qui ont ouvert des dossiers pour que les différentes personnes concernées puissent s'exprimer. Il ressort de ces dossiers qu'il faut briser le carcan de l'option sécuritaire, ou au moins ne pas s'en satisfaire. La lecture de certains articles permet de discerner les différents paramètres de la question (individus, doctrine, langue, etc.), en elle-même et dans ses prolongements (structure et cohésion de la société). Le fait que les aspects sécuritaires l'aient emporté est un des facteurs qui rendent chroniques ces événements. Il est impossible d'en sortir de la sorte. D'après nous, le dialogue, pour autant qu'on en suive les règles de base, permettrait peut-être d'identifier le nœud du problème ou, à tout le moins, de mettre en évidence les différentes tendances en présence. L'observation attentive est sans doute une étape nécessaire à l'identification de multiples facteurs mêlés. Dans cette question qui revêt une importance particulière, il ne faut pas uniquement se limiter à la relation qu'entretiennent le pouvoir et les éléments extrémistes et terroristes. En établir les fondements culturels et intellectuels sera peut-être utile dans la définition des moyens appropriés pour y remédier. 


\section{NOTES}

1. L'Annuaire de l'Umma, présentation et traduction de F. Burgat et B. Dupret, Égypte/Monde arabe $\mathrm{n}^{\circ} 11, \mathrm{pp} .183-192$.

2. Al-Umma fi 'âm. Taqrîr hawli 'an al-chu'ûn al-siyâsiyya wa-l-iqtisâdiyya al-'arabiyya (La Nation en un an. Rapport politique et économique arabe), Dâr al-tabâ'a wa-l-nachr al-islâmiyya, Le Caire, 1993.

3. Assistés de 'Abd al-Wahâb al-Masîrî, Nadia Mustafâ, Suhayr al-Ma'tûf, Sayf al-Dîn 'Abd alFattâh, Hamdî 'Abd al-Rahmân, Ibrâhîm al-Bayyûmî Ghânim et Muhammad Chawq.

4. F. Burgat et B. Dupret, op. cit.

5. «Comme c'est la publicité qui assure la santé financière d'un quotidien et non ses ventes, le régime parvient, en faisant pression sur les annonceurs, à limiter efficacement la capacité d'expression de l'opposition. Dans le cas d'Al-Cha'b, le journal est vendu à perte. L'autre limite, beaucoup plus significative, tient à ce que la liberté de la presse est circonscrite aux supports écrits et - eu égard au taux d'analphabétisme - ne touche qu'une faible partie de la population » (F. Burgat avec la collaboration de B. Dupret, "Cacher le politique. Les représentations de la violence en Égypte ", Maghreb-Machrek, 142, 4/1993, p. 41).

6. ROY O., L'échec de l'islam politique. Le Seuil (coll. Esprit), Paris, 1992.

7. Voir, s'agissant de cette forme de censure, B. Botiveau : «Penser, dire, interdire. Logiques et enjeux de la censure des écrits en Égypte », Égypte/Monde arabe 14, 4/1993, pp. 133-162.

8. Ainsi, parmi tant d'autres exemples, le livre de Rif'at Sayyid Ahmad, Al-Nabî al-musallah (Le Prophète armé), 2 tomes, Londres, Riyâd al-Rayyis li-l-kutub wa-l-nachr, 1991, ou la publication périodique de la Jama'a islamiyya, Kalimat haqq (Parole de vérité), sans références.

9. Ainsi la version anglaise du livre de F. Burgat, The Islamic Movement in North Africa, Texas University Press, 1993, qui, après avoir été admise une première fois à la librairie de l'Université américaine $\mathrm{du}$ Caire, s'est vu refuser l'entrée en Égypte la seconde fois. Ainsi en va-t-il probablement aussi des livres de J. S. Esposito, introuvables sur le marché égyptien du livre anglais.

10. Voir, à ce sujet, la communication de Hasanayn Tawfîq Ibrâhîm lors des cinquièmes rencontres franco-égyptiennes de science politique, à paraître dans les Dossiers du CEDEJ.

11. L'on peut mettre cette remarque en parallèle avec celle d'A. Roussillon qui évoque "une sorte de partage du travail entre les islamistes, laissés libres d'islamiser à leur guise leur environnement en contrepartie des services sociaux qu'ils assurent en palliant les déficiences de l'État, et les autorités locales, assurant la médiation entre les jamâ'ât et le pouvoir central tout en préservant les apparences du maintien de l'ordre républicain» («Changer la société par le jihad", à paraître dans les Actes des cinquièmes rencontres franco-égyptiennes de science politique, Dossiers du CEDEJ, 1994).

12. Tel a d'ailleurs été l'objet de l'intervention de 'Isâm al-'Aryân, lors de la séance du colloque de discussion du Rapport stratégique consacré aux développements politiques en Égypte (24 novembre 1993), selon lequel assimiler les Frères musulmans et les jamâ'ât islamistes radicales relevait quasiment de la diffamation. Ce à quoi Usamâ Ghazalî Harb, représentant du Centre d'études stratégiques, répondit que la revendication du même projet politique par l'ensemble des tendances de l'islamisme était suffisante pour les associer dans le même chapitre. 
INDEX

Mots-clés : annuaire de l'Umma, Fawda (Farag), Frères musulmans, géopolitique

\section{AUTEUR}

\section{BAUDOUIN DUPRET}

CERMAC, Université de Louvain 